\title{
Truncated singular value decomposition in ripped photo recovery
}

\author{
Kong Hoong Lem $^{1 *}$ \\ ${ }^{1}$ Faculty of Science, Universiti Tunku Abdul Rahman, Jalan University, Bandar Barat, 31900 \\ Kampar, Perak, Malaysia
}

\begin{abstract}
Singular value decomposition (SVD) is one of the most useful matrix decompositions in linear algebra. Here, a novel application of SVD in recovering ripped photos was exploited. Recovery was done by applying truncated SVD iteratively. Performance was evaluated using the Frobenius norm. Results from a few experimental photos were decent.
\end{abstract}

\section{Introduction}

Singular value decomposition is a matrix decomposition that decomposes or factorizes a matrix as a product of three component matrices. It was established ways back in the 19th century due to the contributions by Eugenio Beltrami, Camille Jordan, James Joseph Sylvester, Erhard Schmidt, and Hermann Weyl [1].

Singular value decomposition always appears in problems that entail analysis of large amount of data because data often comes in the form of matrix. The application of SVD has been well known in principal component analysis, data and image compression, and in solving least square problems via pseudoinverse [2-4]. Its application is very board applicable to a large scope of problems especially when the problems are representable in matrix and involve matrix approximation. Other SVD applications have been found in areas such as dynamical systems, genomics, textual searching, robotics, financial mathematics and in many others. Interested readers may refer to, for example, [5] and references therein. However, application of truncated SVD in ripped photos recovery is not widely reported in the literature.

In the literatures, ripped recovery was commonly treated as missing data interpolation. The pixel values on the ripped regions were considered lost, so recovery was done by filling in the values using the embracing data. Various professional filling strategies had been reported, for examples, in [6-9]. The objective of this study is to highlight the missing data treatment under the SVD paradigm which is simpler though not as much robust.

\section{The proposed approach}

In computer, grey scale digitized photos are treated as a matrix of pixel values called the image matrix. Every entry of the image matrix stores the grey level of a pixel which floats

*Corresponding author: lemkh@utar.edu.my 
in the interval $[0,1]$. This fact makes matrix operations, such as SVD, play a role in photo processing. Prior to the exposition of the iterative truncated SVD ripped recovery approach, a very brief description on SVD is given.

\subsection{The SVD}

For a given matrix $A$ of size $m \times n$, the singular value decomposition of $A$ is a factorization

$$
A=U S V^{T}
$$

where $U$ is $m \times m, S$ is $m \times n$, and $V$ is $n \times n$. Both $U$ and $V$ are orthogonal matrices while $S$ is diagonal and its diagonal entries $\sigma_{i}$ are known as the singular values of $A$. The singular values are positive and arranged in descending order such that $S=\operatorname{diag}\left(\sigma_{1}, \sigma_{2}, \ldots, \sigma_{n}\right)$ and $\sigma_{1} \geq \sigma_{2} \geq \cdots \geq \sigma_{n} \geq 0$. The square of $\sigma_{i}$ are the eigenvalues of $A A^{T}$ (and $A^{T} A$ ). For a matrix of rank $r, S$ will have $r$ leading nonzero diagonal entries. The columns of $U$ and $V$ are called the left and right singular vectors of $A$ and are the eigenvectors of $A A^{T}$ and $A^{T} A$ respectively. The column $\boldsymbol{u}_{i}$ of $U$, the column $\boldsymbol{v}_{i}$ of $V$, and the singular values $\sigma_{i}$ of $A$ stisfy

$$
A \boldsymbol{v}_{i}=\sigma_{i} \boldsymbol{u}_{i}
$$

Sometimes, it is useful to regard equation (1), the SVD of $A$, as an outer product expansion (somewhat analogous to series expansion) of $A$ in terms of the singular values and the columns of $U$ and $V$ :

$$
A=\sum_{i=1}^{r} \sigma_{i} \boldsymbol{u}_{i} \boldsymbol{v}_{i}^{T}
$$

Here $r$ is the rank of the matrix $A$ and is defined as the dimension of the column space of $A$. In this context, $r$ can be regarded as the number of nonzero singular values of $A$.

\subsection{The truncated SVD and the low-rank matrix approximation}

The $k$-truncated SVD of a matrix $A$ is done by keeping only the first $k \leq r$ nonzero singular values (and hence the first $k$ columns of $U$ and $V$ ) such that

$$
A_{k}=\sum_{i=1}^{k} \sigma_{i} \boldsymbol{u}_{i} \boldsymbol{v}_{i}^{T} \approx A
$$

The resultant matrix $A_{k}$, known as the rank- $k$ approximation to $A$, is just an approximation of the matrix $A$ since $(r-k)$ nonzero singular values are discarded.

Since SVD yields descending singular values, the truncated SVD, which only packs the first few largest singular values, will give an approximation that encapsulate the most essential weightage.

\subsection{The iterative truncated SVD ripped recovery algorithm}

The main function in ripped recovery is to dissipate the tear lines meanwhile maintain the natural look of the photos. As a pre-process, the tear lines on the digitized ripped photo were 
manually erased. These erased regions were recognized as the flaw region and their location were stored. Thus the image matrix consists mostly the perfect region and a minor portion of flaw region such that $A=A$ (perfect) $\cup A$ (flaw). The iterative truncated SVD recovery idea is described in the following algorithm.

Step 1: The image matrix $A$ undergoes a $k$-truncated SVD to obtain its low-rank approximation $A_{k}$. As in $A$, there are perfect and flaw regions in $A_{k}$ also such that $A_{k}=A_{k}$ (perfect) $\cup A_{k}$ (flaw).

Step 2: The matrix entries of $A$ within the flaw regions are all updated by their corresponding approximated entries of $A_{k}$ while the rest of the entries of $A$, in the perfect regions, are kept unchanged. This produces $\tilde{A}=A$ (perfect) $\cup A_{k}$ (flaw).

Step 3: The Frobenius norm of $A-\tilde{A}$ is computed to check against the preset tolerant $\varepsilon$. If $|A-\tilde{A}|_{F}>\varepsilon$, then $A$ is replaced by $\tilde{A}$ and go back to Step 1; else proceed to the next step.

Step 4: Output $A$.

In each iteration, the low rank approximation of the previous image matrix is computed. Due to low rank approximation, the pixel value in the flaw region would spontaneously blend with the surrounding pixel values coming from the flawless region. This kind of iterative blending created certain interpolation effect that made the tear lines faint away. And during the process, the truncated SVD that was capturing the most significant characteristics of the photo would infer the pixel value to restore the photo.

\subsection{The performance measure and the Frobenius norm}

As an objective performance measure, the grey level differences between the undamaged photos (assume available here) and their recovered versions were computed by adopting the Frobenius norm. The Frobenius norm of a $m \times n$ matrix $A$ is defined by

$$
\|A\|_{F}=\sqrt{\sum_{i=1}^{m} \sum_{j=1}^{n}\left|a_{i j}\right|^{2}}
$$

It is in fact the square root of the sum of the absolute squares of all its entries.

To estimate how good the recovery is, the Frobenius-based similarity level $L_{F}$ between the undamaged and the recovered image matrices is defined

$$
L_{F}=1-\frac{\left\|A_{0}-\tilde{A}(s)\right\|_{F}}{\left\|A_{0}-A_{\text {rip }}\right\|_{F}}
$$

where $A_{0}$ is the image matrix of the undamaged photo, $A_{\text {rip }}$ is the image matrix of the ripped photo, $\tilde{A}(s)$ is the image matrix of the recovery after $s$-th truncated SVD iterations. The fraction in the equation ( can be thought off as the relative error. For a perfect recovery, $\tilde{A}(s)=A_{0}$, there will be no difference between the recovery photo and the undamaged photo. Hence generally, the closer the value of $L_{F}$ to unity, the better the recovery will be. 


\section{Experiments and discussion}

The proposed iterative truncated SVD approach was experimented on three test photos. All the test photos in this study were "synthetic" such that their undamaged versions (before ripped) were available. The three test photos were arbitrarily chosen to be (i) portrait, (ii) scenery and (iii) fruits. Only grey scale photos were considered here.

To show the recovery capability of the iterative truncated SVD approach, the undamaged, the ripped, and the recovered versions of the three test photos, lined up from left to right in series, were shown in Fig. 1, Fig. 2, and Fig. 3, one after another.
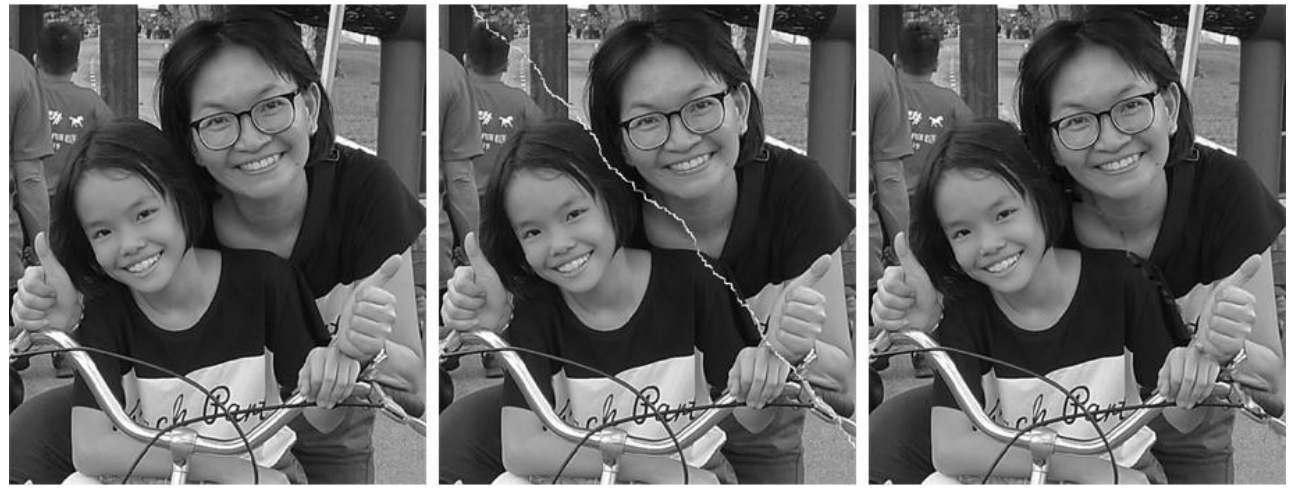

Fig. 1. The first test photo (portrait): the undamaged, the ripped, and the recovered versions were arranged from left to right in series.
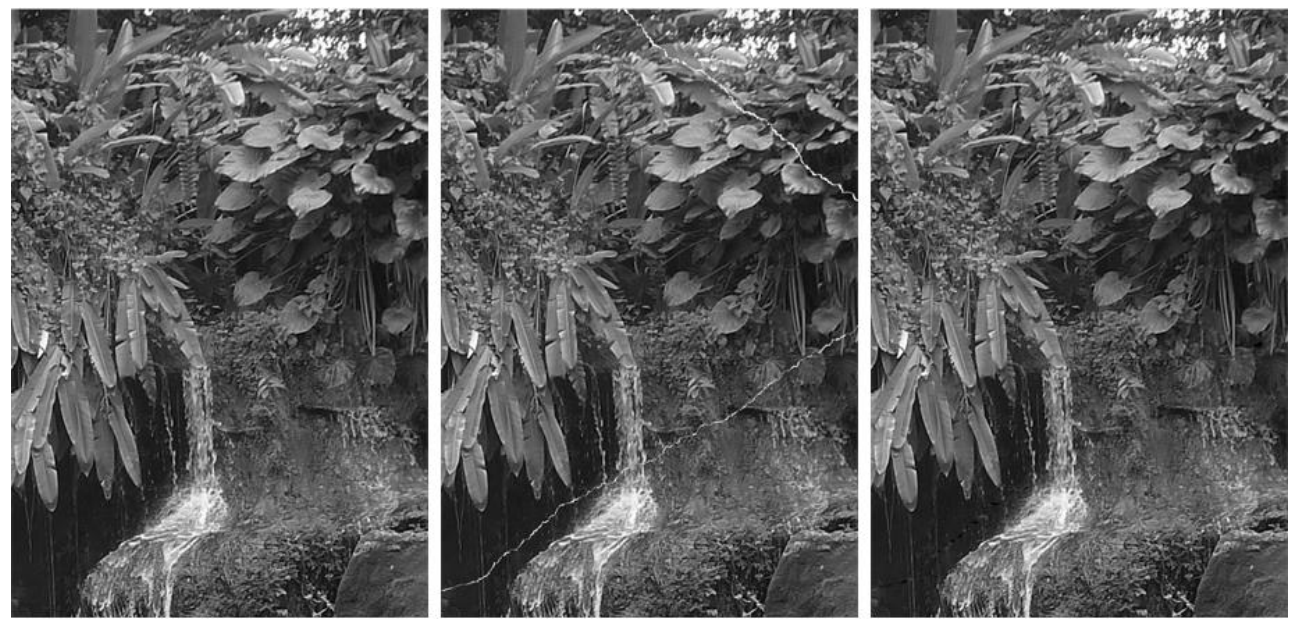

Fig. 2. The second test photo (scenery): the undamaged, the ripped, and the recovered versions were arranged from left to right in series. 

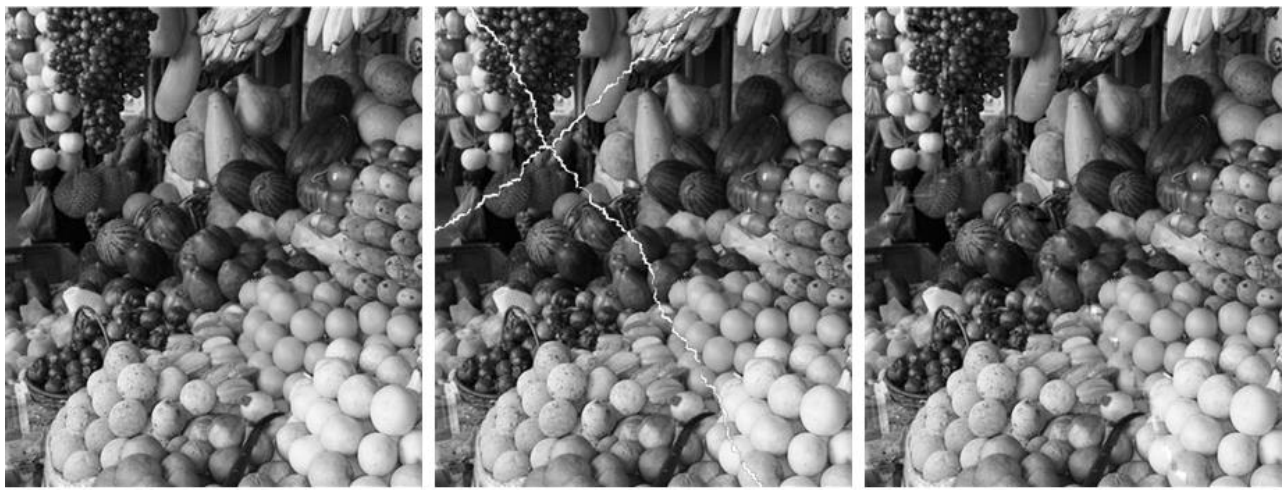

Fig. 3. The third test photo (fruits): the undamaged, the ripped, and the recovered versions were arranged from left to right in series.

In every iteration during the truncated SVD process, the recovery progressed gradually. As a sample, the changes in the third test photo (fruits) during the first few iterations were displayed in Fig. 4.
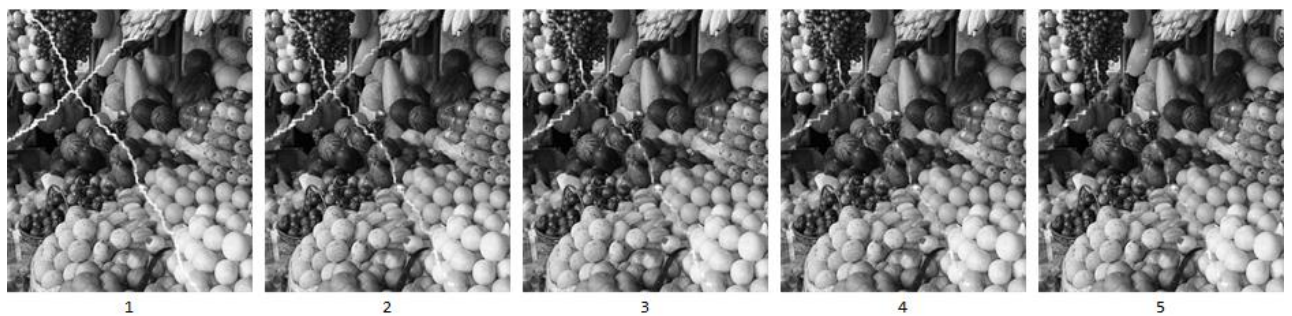

Fig. 4. The changes in the third test photo (fruits) as the truncated SVD iteration progressed.

Though the iterative truncated SVD managed to make the tear lines to be less obvious, the recoveries were not seamless. Artifact was still noticeable especially under a large zoom in.

\subsection{The similarity level}

The Frobenius-based similarity level as defined in equation (6) was computed for each test photo and was listed in the following table.

Table 1. The Frobenius-based similarity level for each test photo.

\begin{tabular}{|c|c|}
\hline Photo & Similarity level \\
\hline Portrait & 0.627922 \\
\hline Scenery & 0.704459 \\
\hline Fruits & 0.643498 \\
\hline
\end{tabular}

In fact, the Frobenius-based similarity level is not a practical assessment because the undamaged photo must be available for its calculation. So, there will be no qualitative measure in real application, it all depends on naked eye observation to determine how natural looking the recovery is. 


\section{Conclusion}

Apart from aesthetic appeal, ripped photo recovery may be meaningful when the photo has great historical value. Besides, recovering ripped photos may play a major role helping crime inspection in forensics. An interesting application of SVD in ripped photo recovery experimented on three samples had been presented here. The algorithm of the iterative truncated SVD was rather simple yet it produced encouraging visual restoring quality which was slightly to see. Again, this seems another manifestation that SVD is ubiquitous in the area of image processing.

\section{References}

1. G. Stewart, SIAM Rev. 35, 4, 551-566 (1993)

2. D. Kalman, College Math. J. 27, 1, 2-23 (1996)

3. G. Strang, Linear algebra and its applications, $4^{\text {th }}$ ed. (Thomson Brooks/Cole, 2006)

4. D. C. Lay, Linear algebra and its applications, $3^{\text {rd }}$ ed. (Pearson, 2006)

5. C. D. Martin, M. A. Porter, Amer. Math. Monthly 119, 10, 838-851 (2012)

6. R. Bornard, E. Lecan, L. Laborelli, J. -H. Chenot, Missing data correction in still images and image sequences, Proceedings of the tenth ACM international conference on Multimedia, 355-361(2002)

7. A. Rares, M. Reinders, J. Biemond, IEEE Trans. Image Process. 14, 10, 1454-1468 (2005)

8. M. Bertalmio, G. Sapiro, V. Caselles, C. Ballester, Image inpainting, in Proceedings of the 27th annual conference on Computer graphics and interactive techniques SIGGRAPH'00, 417-424 (2000)

9. A. Kokaram, S. Godsill, IEEE Trans. Signal Process. 50, 2, 189-205 (2002) 